\title{
AbORDAJES TEÓRICOS SOBRE LAS JUVENTUDES INDÍGENAS EN LATINOAMÉRICA
}

\author{
Theoretical Approaches to Indigenous Youth in Latin America
}

\author{
Laura Kropff-Causa \\ Valentina Stella
}

\begin{abstract}
Resumen: Una de las dificultades al proponer una sistematización de la información sobre juventud indígena en Latinoamérica tiene que ver con que las categorías "juventud" e "indígena" no emergen como datos directos de la realidad, sino que son categorías construidas a partir de definiciones hegemónicas que resultan de la confluencia de diferentes agencias. Para contribuir al análisis de este entramado, esta investigación se focaliza en las definiciones teóricas con las que la academia latinoamericana ha contribuido en los últimos años. Finalmente, se propone el abordaje de edad y etnicidad como clivajes que definen la movilidad estructurada de subjetividades, identidades y agencias cuya trama debe leerse situada en cada contexto sociopolítico e histórico particular.
\end{abstract}

Palabras clave: edad, etnicidad, subjetividades, categorías sociales.

Abstract: One of the main difficulties of organizing information about indigenous youth in Latin-America is that neither "youth" nor "indigenous" are categories that represent a factual reality but are constructed based on hegemonic definitions that result from the confluence of different agents. This essay considers the ways in which Latin-American scholarship has contributed to this construction in the last years. Finally, the article offers an alternate approach that uses "age" and "ethnicity" as cleavages to define movement along diverse continuums of subjectivity, identity and agency. Throughout, we argue that these frameworks must be al ways read as situated in their particular sociopolitical and historical context.

Keywords: age, ethnicity, subjectivities, social categories.

\footnotetext{
Laura Kropff Causa. Doctora en Ciencias Antropológicas por la Universidad de Buenos Aires. Investigadora adjunta del Consejo Nacional de Investigaciones Científicas y Tecnológicas (CONICET) en el Instituto de Investigaciones en Diversidad Cultural y Procesos de Cambio (IIDyPCa), dependiente del CONICET y de la Universidad Nacional de Río Negro (UNRN), Argentina. Temas de especialización: antropología de la edad, estudios étnicos, antropología política, antropología histórica. Correo electrónico: kropff@unrn.edu.ar.

Valentina Stella. Licenciada en Ciencias Antropológicas por la Universidad de Buenos Aires. Becaria doctoral del Consejo Nacional de Investigaciones Científicas y Tecnológicas (CONICET) en el Instituto de Investigaciones en Diversidad Cultural y Procesos de Cambio (IIDyPCa), Argentina. Temas de especialización: estudios étnicos, antropología de la memoria, antropología política. Correo electrónico: vstella@unrn.edu.ar.
}

Enviado a dictamen: 7 de abril de 2016. Aprobación: 7 de septiembre de 2016. Revisiones: 2. 


\section{Introducción}

L a idea de escribir este artículo surgió de la necesidad de sistematizar la producción dispersa en la que se abordan problemáticas ubicadas en la intersección entre juventud y etnicidad en Latinoamérica. El cruce de la cuestión étnica con la cuestión juvenil ha sido reiteradamente declarado área de vacancia en foros de intercambio académico y, en función de ello, en la segunda década del siglo XXI la tendencia comenzó a cambiar. ${ }^{1}$

Una de las dificultades más notables al momento de proponer una sistematización de la información sobre juventud indígena en Latinoamérica tiene que ver con que las categorías "juventud" e "indígena" no emergen como datos directos de la realidad, sino que son categorías construidas como dato a partir de definiciones hegemónicas que resultan de la confluencia asimétrica de agencias diferentes como los estados nacionales, los organismos multilaterales o las organizaciones no gubernamentales. Las articulaciones de agencia que se fundan en el posicionamiento en tanto "jóvenes indígenas" participan de esta construcción y se ven en la obligación de producir sentido sobre las interpelaciones, ya sea refutándolas o refrendándolas. De allí que devenga necesaria la deconstrucción de estas categorías (Pérez Ruiz, 2011).

En ese marco, la academia misma se constituye como agencia con un capital simbólico propio que se pone en juego al momento de legitimar criterios para definir/nominar lo que se entiende por "juventud indígena". Por ello, en función de contribuir al análisis de ese complejo entramado, en esta investigación se coloca el foco en las concepciones teóricas con las que la academia latinoamericana ha influido en esta arena en los últimos años. ${ }^{2}$ Pretendemos, por un lado, poner en valor y analizar críticamente los trabajos de investigación que se han hecho en el área y, por otro lado, contribuir a la discusión teórica y política que el trabajo en tal área demanda.

Para mapear las definiciones presentes en las producciones dispersas en las que se analizan juventud y etnicidad nuestra estrategia fue, en primer lugar, compilar las ponencias presentadas en foros de discusión sobre la temática en países latinoamericanos. Así, incorporamos al corpus los aportes realizados por distintos investigadores e investigadoras en las sucesivas reuniones del Congreso Argentino de Antropología Social (2004, 2006, 2008 y 2011), en los simposios vinculados al tema en el 53 Congreso de Internacional de Americanistas (México, 19 al 24 de julio de 2009) y en el Coloquio Internacional "Juventud, etnicidad, ruralidad y movimientos translocales en Latinoamérica" organizado en Bariloche en el año 2010. ${ }^{3}$ Paralelamente, realizamos una compulsa bibliográfica en revistas, bibliotecas y sitios académicos de internet. Como resultado de ese cotejo, el corpus analizado aquí está compuesto por artículos, ponencias y tesis en los que se analizan casos en Argentina, Bolivia, Brasil, Chile, Colombia, Ecuador, Guatemala, México y Perú. Sólo encontramos publicaciones unitarias centradas en el tema en Chile y en México. ${ }^{4}$ Dada la dispersión de las producciones, es probable que haya aportes a los que no logramos acceder, por lo que no suponemos que este mapeo agote todas las investigaciones referidas al tema. No es nuestra intención construir un estado del arte que contemple todos los problemas y subáreas temáticas en torno a la juventud indígena, ${ }^{5}$ sino elaborar una primera aproximación a los enfoques teóricos.

Organizaremos esta exposición a partir de cuatro énfasis teóricos generales que nos permiten agrupar los distintos enfoques que conforman nuestro corpus. En el primer apartado presentaremos perspectivas que toman la condición etaria y la identidad étnica como puntos de partida para tratar, desde allí, problemas que afectan a los sujetos definidos desde esas categorías. En el segundo apartado introduciremos un conjunto de abordajes en los que se considera la juventud como dato biológico/cronológico y se construye la etnicidad como problema. En el tercer apartado se reúnen investigaciones basadas en la definición de la etnicidad como dato -fundado en contenidos culturales o en estadísticas nacionales/estatales- y en las que se problematiza la juventud. Finalmente, en la última sección incluiremos aportes que analizan tanto edad, como etnicidad. Entre ellos, incluimos nuestros propios 
abordajes, que parten de entender la edad y la etnicidad como "clivajes", es decir, como líneas que estructuran la organización social de identidades, subjetividades y agencias estableciendo dinámicas de agregación/ desagregación (Briones y Siffredi, 1989). ${ }^{6}$

\section{Juventud e identidad étnica como datos dados}

Las perspectivas incluidas en este enfoque general se pueden organizar en tres subgrupos. En primer lugar, algunas investigaciones definen la juventud indígena a partir de datos demográficos y otras estadísticas. En segundo lugar, encontramos análisis que consideran a la juventud indígena como lugar de enunciación y analizan los discursos y las producciones estéticas que se generan desde allí. Finalmente, hallamos trabajos que se fundan en la etnografía situada con comunidades indígenas o grupos étnicos en contextos rurales y urbanos. Estos trabajos tienen en común el hecho de que ni las categorías etarias ni las étnicas constituyen objeto de reflexión, sino punto de partida para abordar otros temas.

En el caso de los estudios que se fundan en estadísticas, los temas más recurrentes están atravesados por la pregunta sobre la integración en la sociedad mayor del sector recortado para el análisis. Los tratamientos de problemas en torno a la educación, el trabajo, la salud o la pobreza tienen en común su argumentación a favor del desarrollo de políticas públicas específicas orientadas al sector. En esa línea, encontramos investigaciones que comparan la situación de los jóvenes indígenas con el resto de la población en cuanto a temas como pobreza, trabajo, educación, políticas de salud, organización política o derechos humanos en Argentina (Padawery Rodríguez Celín, 2015, entre otros), Chile (Candia, Damianovic y Leibovitz, 2006; Castro, Rojas y Ruiz, 2008, entre otros), Colombia (Alvarado, Loaisa y Patiño, 2010; Mayorga y Marcia, 2010, entre otros), Ecuador (Llanos, 2013), México (Rodríguez, 2015; Igreja, 2008; Pacheco, 2009; Pérez Ruiz, 2008b; Valladares, 2008), Perú (De Rivero, 2009) y en América Latina en general (Del Popolo, Oyarce y Ribotta, 2010).
Las aproximaciones que se fundan en la definición de la juventud indígena como lugar de enunciación colocan el foco en el campo de las producciones estéticas o artísticas y, específicamente, en la música. Los análisis se centran en las producciones musicales generadas por jóvenes indígenas en contextos comunitarios, nacionales y globales. Las interpretaciones tienen que ver con las características de los géneros musicales, las performances y las estéticas que combinan anclajes etarios y étnicos. Las preguntas se relacionan con los efectos que, en tanto prácticas culturales, tienen a nivel de los roles comunitarios o de las identidades en la tensión entre tradición y modernidad que se genera en el marco de procesos vinculados a la globalización.

En este conjunto se incluyen investigaciones sobre cinco casos. Una de ellas trata sobre la resignificación de la música sound entre jóvenes aymara en Chile (Guerrero, 2007), otra revisa la apropiación de la música hip-hop entre grupos de jóvenes indígenas de distintos puntos del continente americano -Bolivia, Chile, Ecuador y México- (Bojórquez, 2009), otra analiza el movimiento de rap político y la apropiación del hip hop en el altiplano boliviano (Kunin, 2009) y la cuarta se centra en las performances musicales religiosas de los jóvenes toba en el Chaco argentino (Citro, 2005).?

Aunque los cuatro trabajos comparten el recorte del objeto y algunas preguntas centrales, hay énfasis específicos que vale la pena destacar. Tanto Bernardo Guerrero Jiménez como Tiosha Bojórquez Chapela entienden que las apropiaciones de géneros musicales que provienen de circuitos globales implican la recuperación de diversos elementos tradicionales para recontextualizarlos dentro de lo que Bojórquez Chapela define como "marco transcultural" (2009: 249). Estas prácticas devienen, entonces, en registros para pensar los fenómenos de la globalización sin considerarlos como una amenaza para la preservación de las identidades indígenas, sino como oportunidades de reactualización y refuncionalización creativa.

El énfasis del trabajo de Johana Kunin es diferente, ya que la autora está interesada en el contexto político nacional. Analiza los circuitos de producción, distribución y consumo de rap político en el altiplano 
boliviano entendiéndolos comoun movimiento artístico/ político que participa del proceso de reconstrucción discursiva y subjetiva de la identidad aymara y boliviana contemporánea. ${ }^{8}$ Para Kunin, no se trata de una mezcla cultural simple entre tradición y modernidad globalizada, sino que la "hibridez" está mediada por relaciones de poder, hegemonía y resistencias situadas en contextos nacionales y locales. Por su parte, Silvia Citro se centra en el contexto comunitario para analizar el modo en que los jóvenes toba usan estratégicamente la música como recurso cultural polifacético para legitimar su lugar social en la comunidad y para proyectarse en contextos más amplios atravesados por "relaciones interculturales". Si los primeros dos autores analizan el contexto global, la tercera retoma el contexto nacional y la cuarta el contexto comunitario.

En el último subgrupo de estudios basados en los anclajes etarios y étnicos como punto de partida, encontramos una serie de trabajos fundados en la etnografía situada. ${ }^{9}$ Desdeesteenfoque, lasinvestigaciones relevadas se centran en la migración, la experiencia de jóvenes indígenas en contextos urbanos, la participación en organizaciones políticas, etcétera. Basándose en el análisis de estos temas, los autores y autoras vuelven sobre una de las preguntas clásicas de la antropología, la que indaga en la tensión entre cambio y continuidad (Pérez Ruiz, 2008). En este conjunto de trabajos se analizan casos de jóvenes manchineri, kaxinawá y apuriña en contextos urbanos de Brasil (Virtanen, 2007), jóvenes quechua en Bolivia (Terrazas, 2008), jóvenes indígenas en las ciudades mexicanas (Pérez Ruiz, 2008; Solís y Martínez, 2015; Serrano, 2015), jóvenes williche de Chiloé (Muñoz, 2006) y jóvenes indígenas de Magallanes (Aguilera y Flores, 2006) en Chile.

Desde una perspectiva fundada en conceptos de Pierre Bourdieu - por ejemplo, campo y habitus - Pirjo Kristina Virtanen, Daniel Solís, Consuelo Martínez y María Laura Serrano Santos sostienen que la identidad de los jóvenes indígenas en las ciudades se configura de manera contextual y está en permanente cambio. No es resultado de un retorno a las raíces ni de la integración total a la vida urbana, sino que emerge de entrecruces dinámicos. ${ }^{10}$
Desde una perspectiva teórica distinta, Maziel Terrazas Merino sostiene que los bienes e imaginarios culturales propios de la globalización que ingresan en el ayllu Chari (Bolivia) a través de los jóvenes quechuas, afectan el estilo de vida, el imaginario y la identidad colectiva socavando las pautas tradicionales. Por su parte, Maya Lorena Pérez Ruiz afirma que los jóvenes indígenas que viven en las ciudades mexicanas atraviesan tanto "procesos de pérdida cultural y de abandono de identidades propias", como de reafirmación identitaria (2008: 64). Asimismo, la autora señala que es necesario disociar "identidad de cultura", y establece que no hay una relación directa entre cambio cultural y pérdida de identidad.

Dentro del mismo enfoque, fundado en la etnografía situada, se encuentra el trabajo de Manuel Muñoz Millalonco, quien analiza los conflictos intergeneracionales entre los williche de Chiloé. El autor se centra, por un lado, en los reclamos mutuos entre ancianos y jóvenes en torno a la tradición y a la lucha por el territorio y, por otro, en la participación política de los jóvenes en relación con el Estado y con las comunidades. Nelson Aguilera Aguila y Blas Flores Aguila colocan el foco en la participación política, pero los autores señalan con preocupación la desvinculación de los jóvenes de las organizaciones indígenas de Magallanes interpretándola como efecto de la poca claridad y la falta de legitimidad de las organizaciones. ${ }^{11}$

\section{Juventud como dato, identidad étnica como problema}

El problema central que concita el interés de los investigadores que toman la juventud como dato y que problematizan la etnicidad es la presencia de jóvenes indígenas en contextos urbanos. Nuevamente encontramos trabajos que toman el caso de los kamaiura (Brasil), a los que se suman investigaciones sobre los mapuche (Chile), jóvenes wixaritari (huicholes), en México y sobre jóvenes indígenas —en términos genéricos- de Ecuador y Guatemala. En cuanto al enfoque teórico, identificamos dos tendencias 
contrapuestas que, siguiendo a Briones (1998), podemos definir como enfoques sustancialistas y formalistas.

En primer lugar, hallamos autores y autoras que parten de una concepción sustancialista de la etnicidad. ${ }^{12}$ Desde esta perspectiva, lo juvenil se contrapone a la etnicidad porque se identifica como opuesto a la tradición. El problema central, entonces, tiene que ver con el cambio y la continuidad. Se identifica a la juventud como agente de transformación orientado hacia lo urbano y a la etnicidad como efecto de una serie de contenidos culturales territorializados en áreas rurales que son defendidos por los mayores de la comunidad. En esta línea de pensamiento, la etnicidad se ve desafiada por la juventud en tanto esta última se identifica como motor de aculturación o transculturación. El problema es, entonces, la pérdida de contenidos culturales definidos como tradicionales.

En esta línea, el trabajo de Taciana Vaneska Vitti y Carmen Junqueira se centra en la etnografía de las comunidades kamaiura y pone el foco en los efectos de las migraciones de los jóvenes a la ciudad. El contexto urbano representa el primer contacto con los bienes y valores del capitalismo que se incorporan a través, por ejemplo, de prácticas de consumo. ${ }^{13}$ Las autoras señalan que la etnicidad - definida a partir de una serie de contenidos culturales territorializados en las comunidades - está desafiada por las prácticas de los jóvenes en su vinculación con espacios urbanos (Vitti y Junqueira, 2015).

En segundo lugar, encontramos autores que parten de una definición "formalista" de la etnicidad. ${ }^{14} \mathrm{Al}$ abandonar la perspectiva sustancialista, se cuestiona la asociación directa entre etnicidad y ruralidad y se pone el foco en las resignificaciones de las identidades étnicas en contextos diferentes (Aravena, 2006). Los trabajos que se incluyen en esta línea abordan la situación de los jóvenes en relación con los contextos urbanos, pero, en lugar de pensarlos como agentes de cambio opuestos a la tradición, los ven como agentes activos que desarrollan estrategias para redefinir y legitimar su pertenencia étnica. En esta perspectiva se inscribe el trabajo de Clorinda Cuminao Rojo (2006) sobre jóvenes mapuche en Santiago (Chile), el de Alexis Rivas Toledo (2008) sobre jóvenes indígenas en Ecuador, el de Manuela Camus (2008) sobre la frontera étnica en Guatemala, el de Marta Romer (2008) sobre jóvenes indígenas en la Ciudad de México y el trabajo de Diana Negrín da Silva (2015) sobre jóvenes wixaritari (huicholes) que estudian y trabajan en Tepic y Guadalajara (México).

\section{Identidad étnica como dato, juventud como problema}

El tercer conjunto de trabajos toma la identidad étnica como dato y construye la edad -y la juventud en particular - como problema. En este grupo de investigaciones encontramos dos lecturas diferentes. La primera se basa en la discusión teórica sobre el concepto de juventud, en especial sobre las connotaciones etnocéntricas que subyacen a la definición y que invisibilizan las subjetividades, identidades y agencias de los jóvenes indígenas. En esta línea, encontramos esfuerzos por redefinir el concepto para dar cuenta de casos diferentes. La juventud es, desde este enfoque, objeto de reflexión en tanto problema conceptual para las teorías académicas. Una segunda lectura tiene que ver con la tensión entre teorías nativas y occidentales de la edad. En este caso, el foco está puesto en la relación asimétrica entre las teorías de los distintos pueblos originarios en torno a la edad - que pueden o no incluir una categoría denominada "juventud" - y la teoría occidental promovida por el avance de los Estados nacionales y del capitalismo global sobre las comunidades (Pérez Ruiz, 2008). En el análisis de esta interacción asimétrica hallamos trabajos etnográficos en los que se examinan los procesos dinámicos de negociación, confrontación y transformación de las distintas teorías. Juventud es, en este recorte, un problema en el campo de las teorías sociales.

En la primera perspectiva encontramos el trabajo de Maritza Urteaga (2009), quien, a partir del cuestionamiento a las definiciones etnocéntricas del concepto de juventud, presenta un estado del arte de las investigaciones que visibilizan a las juventudes indígenas rurales y urbanas en México. Por su parte, Luis Fernando García Álvarez (2015) analiza las múltiples formas en 
que se configura la "juventud" en distintos procesos históricos y contextos para determinar la emergencia de un "periodo juvenil" en diferentes grupos, comunidades y asentamientos urbanos étnicos en México. ${ }^{15}$ Uno de los aspectos fundamentales de la conceptualización tanto de Urteaga como de García Álvarez es la heterogeneidad de contextos en los cuales la condición juvenil se pone en juego, demarcando un abanico heterogéneo de trayectorias posibles.

Por su parte, Margarita Calfio Montalvo, cuya investigación se centra en los pueblos originarios de Chile, cuestiona la definición generalizadora de juventud como etapa de transición y coloca el foco en los procesos históricos, políticos, sociales y culturales específicos de cada pueblo en relación con el Estado y con la sociedad mayor en la que se insertan. En esta línea encontramos, también, dos análisis de caso en Brasil y México. El primero es el trabajo de Edmundo Marcelo Mendes Pereira (2011), quien busca entender el significado de la categoría "joven" en un grupo de indígenas terena en Brasil, al tiempo que indaga sobre la experiencia de esos jóvenes en el espacio social urbano. El segundo caso es el surgimiento de juventudes indígenas en el Totonacapan veracruzano que aborda Ariel García Martínez (2012). El autor explica este surgimiento a partir de la interacción de lo que denomina "cultura indígena" y "cultura nacional", que altera los límites simbólicos de las etapas de la vida agraria.

En la segunda perspectiva, que se centra en la relación - muchas veces asimétrica - entre teorías sociales diferentes de la edad, encontramos estudios de caso en Argentina, Bolivia, Brasil, Colombia y México. En estos trabajos el punto de confluencia es la identificación de construcciones occidentales de juventud que interpelan subjetividades que, al mismo tiempo, son interpeladas por otras lógicas de configuración de "alteridades etarias" (Guevara, 2015; Ossola, 2015; Fischer, 2008; Pérez Ruiz, 2008; Zapata y Hoyos, 2005). Las etnografías recuperan, en ese marco, las miradas de los adultos, de los agentes no indígenas (Paladino, 2006; Ruiz Lagier, 2008) y de los sujetos interpelados por estas categorías, es decir, los "jóvenes" (Feixa y González, 2006; Neto, 2004, entre otros).
En su estudio sobre la juventud indígena de Antioquia (Colombia), Carlos Andrés Zapata Cardona y Mauricio Hoyos Agudelo analizan la categoría "juventud" desde la mirada occidental y desde la mirada indígena para luego observar los modos en que opera en diferentes campos de acción como la escuela y los procesos organizativos. En una línea de pensamiento similar, Eva Fischer aborda el caso de los jóvenes aymara de Upinhuaya (Bolivia). La autora asocia las transformaciones en la teoría de la edad con cambios producidos a nivel de las relaciones parentales.

Por su parte, Mariana Paladino trabaja con el pueblo ticuna (Brasil) e indaga sobre la construcción histórica de la categoría "juventud" en relación con teorías nativas de la edad. Según Paladino, la estigmatización asociada a la nueva categoría construye una idea de vulnerabilidad que los adultos y los agentes no indígenas relacionan con procesos de "aculturación" y "pérdida" de un patrón cultural ticuna "tradicional" — aculturación, pérdida y tradición son aquí categorías nativas-.

Con una mirada similar trabaja Joaquim José Neto el caso de los jóvenes del agrupamiento étnico tapuios do Carretão (Brasil), pueblo que se autodefine como descendiente de etnias diversas que resistieron a la colonización —como xavante, caiapó, carajá y javaéy que fueron reunidas en Carretão junto con negros fugitivos de la esclavización. Neto coloca el foco en la perspectiva de los jóvenes tapuio y formula preguntas en torno a la identidad indígena, la demanda de derechos, los proyectos de vida, los adultos y ancianos, la educación, etcétera. Verónica Ruiz Lagier (2008) se hace preguntas similares en el caso de los jóvenes kanjobal de origen guatemalteco residentes en Chiapas, México.

\section{Edad y etnicidad como problemas}

Son pocos los trabajos recopilados en este corpus que problematizan tanto la edad, como la etnicidad. Sin embargo, encontramos en ese conjunto tres tendencias teóricas sutilmente diferentes. La primera de ellas parte de una perspectiva substancialista en términos étnicos $y$, por tanto, sus preocupaciones pasan por los procesos 
de cambio cultural, a la vez que se pregunta qué significa ser "joven" en el marco de esos procesos. En ese sentido, aunque la etnicidad está vinculada a prácticas culturales específicas, la edad se presenta como una experiencia social sobre la que los actores producen significado (Ambriz, 2011). La segunda tendencia busca contextualizar e historizar la emergencia de lo que se denomina el "sujeto indígena joven". En esta perspectiva, juventud y etnicidad serían "condiciones" de un sujeto que debe ser reconocido por los investigadores. Finalmente, la última tendencia entiende que edad y etnicidad son dimensiones que estructuran las prácticas sociales y, por lo tanto, demarcan una cartografía que es necesario reponer.

En la segunda tendencia se enmarca el trabajo de René Unda Lara y Germán Muñoz (2011), basado en una investigación sobre la Sierra Central del Ecuador. Las preguntas iniciales de los autores tienen que ver con lo que significa ser joven en las comunidades indígenas $\mathrm{y}$, en ese sentido, se podría incluir su trabajo entre aquellos que problematizan la edad. Sin embargo, esa problematización se realiza a partir del análisis de las transformaciones históricas en lo que denominan "el espacio social de lo indígena", cuya dimensión fundamental son las prácticas de producción y reproducción vinculadas a la tierra y fundadas en una matriz colonial que se continúa en el contexto republicano. A partir de esa historización se problematiza, también, lo que significa ser indígena. En esta misma tendencia se puede incluir el trabajo de Tania Cruz Salazar (2012) sobre el "sujeto joven indígena" en Chiapas (México), y sobre juventudes indígenas migrantes en California (Estados Unidos) (Cruz, 2015).

En la tercera tendencia se enmarca un conjunto de trabajos que incluye abordajes de la etnicidad, la clase social y la juventud en términos de identidades sociales entramadas (Tipa 2015; Maidana, Colangeloy Tamagno, 2012; Zebadúa, 2009) y perspectivas que recuperan la dialéctica de la interpelación y la articulación de agencia. Uno de los casos que se orienta en el segundo sentido es el artículo de Álvaro Bello Maldonado (2008) sobre los espacios de juventud indígena en una comunidad purépecha de Michoacán (México) que está atravesada por la experiencia migratoria a Estados Unidos. El autor cuestiona las definiciones esencialistas y biologicistas tanto de la edad, como de la etnicidad. Parte de entender que los supuestos sobre los cuales se funda el concepto de juventud no ofrecen alternativas de lectura, e interpreta necesariamente la realidad juvenil indígena como efecto de influencias exógenas y de aculturación. Por otra parte, su definición de etnicidad tiene que ver con los procesos de interacción al estilo formalista. ${ }^{16}$ Edad y etnicidad son, en el trabajo de Bello Maldonado, dos instancias entrelazadas de relación y negociación con profundidad histórica cuya dinámica introduce permanentemente variables de heterogeneización. Consecuentemente, se pueden pensar como "clivajes" (Briones y Siffredi, 1989), lo que lleva a entender que edad, etnicidad, raza, clase o género son clivajes que operan entramados. ${ }^{17}$ El concepto de clivaje -y la posibilidad de pensar clivajes entramados- implica entender que las diferencias entre los grupos no son absolutas, sino relativas, relacionales y contextuales. El análisis del modo en que opera un clivaje en particular puede ser, también, un instrumento metodológico que permita ingresar en la densidad de la trama social por uno de los lugares posibles.

Nuestras propias investigaciones sobre jóvenes mapuche en Argentina se basan en una perspectiva que considera edad y etnicidad como clivajes en el marco de movilidades estructuradas (Grossberg, 1996). Así, edad y etnicidad operan definiendo no sólo lo que otros autores entienden como un sujeto histórico que debe ser re-conocido, sino lo que Grossberg contempla como tres planos de individuación: el de la identidad como construcción temporal de la diferencia, el de la subjetividad como conciencia del tiempo interno y el de la agencia como desplazamiento temporal de la diferencia. Según Grossberg, en la conformación de estos tres planos participan, a su vez, tres tipos de "maquinarias": a) estratificadoras, que regulan el acceso a las experiencias y conocimientos del mundo produciendo subjetividades desiguales, b) diferenciadoras, vinculadas a regímenes de verdad que producen sistemas de identidades y diferencias, 
y c) territorializadoras, que establecen sistemas de circulación entre lugares. Es en el marco de las estructuras sedimentadas de circulación y acceso que resultan de la acción de estas maquinarias, donde se van desarrollando las movilidades en un interjuego entre estabilidad y cambio. Entonces, no se trata únicamente de una distribución desigual de capitales -económicos, sociales, simbólicos-, sino de la disponibilidad diferencial de trayectorias (Briones, 2005). En el caso del problema que nos ocupa, los mapas que definen las trayectorias se constituyen en la intersección entre tramas de relaciones discursivas y de poder organizadas por dos clivajes: la etnicidad y la edad.

En función de analizar la realidad de los pueblos indígenas - según se los define en el campo jurídico-o pueblos originarios - como se los define en el campo de los movimientos sociales-, el concepto de "etnicidad", entendido desde matrices formalistas, presenta algunas limitaciones que tienen que ver con su inespecificidad - ya que se trata de una teoría aplicable a cualquier proceso en el que se negocian y recrean identidades sociales por contraste, y no únicamente a la etnicidadcon la omisión de las relaciones de poder y con la perspectiva sincrónica que no recupera los procesos históricos de relacionamiento. ${ }^{18}$ De allí que partamos del concepto de "aboriginalidad" en tanto construcción de alteridad caracterizada por interpelaciones etnizadas y racializadas que se basa en la presunción de autoctonía de sujetos colectivos en el marco de la consolidación de las matrices que vinculan estados, naciones y territorios (Beckett, 1988). A su vez, la aboriginalidad se articula de forma compleja con el clivaje de clase que organiza la accesibilidad a la experiencia, las identidades/ diferencias y las trayectorias sociales, a partir de la inserción en el sistema productivo en condiciones de desigualdad.

Por su parte, la edad opera comoclivaje estructurando subjetividades e identidades sociales en relaciones asimétricas (Pérez Ruiz, 2008), es decir, generando un sistema de "alteridades etarias" (Kropff, 2011). El capital simbólico de las categorías que definen grados de edad se pone en juego en condiciones de asimetría y opera naturalizando pautas de conducta y haces de roles (Kertzer, 1978) que difieren en distintos contextos geográficos, históricos y sociales. En ese marco, el conflicto entre distintas teorías de la edad tiene que ver con la sedimentación de los sentidos que definen los grados de edad.

Desde esta perspectiva teórica, hemos analizado la configuración de generaciones políticas en el marco del movimiento mapuche contemporáneo en Argentina (Kropff, 2005), así comolaintervención de articulaciones etarias configuradas en el marco del movimiento organizacional en contextos comunitarios y familiares (Stella, 2013). Prestamos atención al modo en que las distintas tradiciones - familiares, organizacionales, mapuche, no mapuche y estatales - puestas en juego, generan un entramado de interpelaciones, articulaciones de agencia y subjetivaciones con diferente grado de sedimentación.

\section{Una pequeña caja de herramientas}

Nuestro objetivo en este texto fue agrupar los trabajos de investigación sobre juventud indígena en Latinoamérica en función de los abordajes teóricos. Organizamos las perspectivas a partir de cuatro grandes enfoques: 1) el que considera edad y etnicidad como datos iniciales, 2) el que problematiza la etnicidad, 3) el que problematiza la edad y 4) el que problematiza tanto edad como etnicidad (ver cuadro 1). A partir de esa organización - basada en intereses propiosrastreamos las herramientas conceptuales que se están utilizando para tratar el tema. Lejos de encontrarnos con un vacío teórico, logramos mapear tanto problemas como conceptos que se van articulando en la medida en que se comienza a consolidar un campo específico de investigación.

Entre los estudios que toman edad y etnicidad como punto de partida, encontramos preguntas sociológicas en torno a la inclusión y la exclusión de los jóvenes indígenas en los sistemas económicos y políticos de distintos países. Los trabajos se basan tanto en datos estadísticos, como en el análisis de políticas públicas y de procesos sociales puntuales. Entre los trabajos que se fundan en este enfoque, encontramos 
también los de autores y autoras que toman como punto de partida la edad y la etnicidad en tanto coordenadas que definen lugares de enunciación o en tanto atributos de grupos en contextos etnográficos situados. Aquí hallamos preocupaciones caras a la antropología, como la indagación en las relaciones entre tradición y modernidad/globalización, y entre cambio y continuidad.

En cuanto al enfoque que se centra en la problematización de la etnicidad, vemos introducida la tensión entre las perspectivas substancialistas y formalistas, que atraviesa el campo de la antropología y de los estudios étnicos. Es, en todo caso, su entrecruce con las definiciones etarias lo que vale la pena profundizar. Si los enfoques substancialistas retoman el problema del cambio y la continuidad y, en relación con esto, analizan procesos que denominan de "aculturación" o "transculturación", los enfoques formalistas se centran en los procesos de "negociación" y "resignificación" de las identidades.

En el conjunto de estudios que problematizan la edad - y la juventud en particular - encontramos aportes específicos orientados a reponer discusiones ya planteadas en el campo de los estudios de juventud. Es especialmente relevante la revisión de las definiciones etnocéntricas de juventud a la luz del contraste entre distintas teorías sociales de la edad. Los estudios que se centran en este campo ponen en evidencia -etnográfica - la arbitrariedad de la edad como dimensión estructurante de las prácticas sociales, así como los efectos situados e históricos de la asimetría entre las distintas teorías en relación con la configuración de subjetividades, identidades y agencias.

Finalmente, el enfoque desde el que se problematiza tanto edad como etnicidad incorpora el desarrollo crítico de los aportes formalistas de los estudios étnicos para pensar las "alteridades etarias". Si la discusión de los estudios étnicos permitió que la antropología dejara de tener por objeto a los indígenas y "su" diferencia para colocar en el foco del análisis la coproducción de etnicidades y nación, este enfoque construye como objeto al proceso mismo de construcción y disputa de alteridades etarias. Como ya se dijo, las construcciones etarias, al igual que las étnicas, son objeto de disputas que generan una arena en la que emergen subjetividades que cargan con interpelaciones diversas. Es la cartografía que define esa arena, la que este último enfoque pretende analizar.

Para concluir, esperamos que este recorrido se convierta en una pequeña caja de herramientas que contribuya a la discusión teórica de los investigadores e investigadoras que comienzan a producir conocimiento sobre la intersección entre edad y etnicidad.

\section{Notas}

${ }^{1}$ A nivel latinoamericano, Carles Feixa y Yanko González Cangas (2006) explican la ausencia de investigaciones sobre identidades juveniles indígenas y rurales a partir del sesgo etnocéntrico presente en el concepto mismo de juventud. Sin embargo, como bien señala Maya Lorena Pérez Ruiz (2011), hacia fines de la primera década del siglo XXI la cantidad de producciones sobre el tema comenzó a crecer.

${ }^{2} \mathrm{El}$ corpus está conformado tanto por autores latinoamericanos como por autores de otros lugares del mundo que participan en foros latinoamericanos de intercambio.

${ }^{3}$ El coloquio, realizado los días 21 y 22 de octubre de 2010, fue organizado por el Instituto de Investigaciones en Diversidad Cultural y Procesos de Cambio (IIDyPCa) con apoyo de la Agencia Nacional de Promoción Científica y Tecnológica y del CONICET. Contó con la participación de investigadores de Argentina, Brasil, Chile, Colombia, Ecuador y México.

${ }^{4}$ Se trata del número 12 de la revista Observatorio de Juventud: "Jóvenes y pueblos originarios" (editada por el Instituto Nacional de la Juventud de Chile en diciembre de 2006), del libro Jóvenes indígenas y globalización en América Latina, compilación de Maya Lorena Pérez Ruiz (editado en 2009 por el Instituto Nacional de Antropología e Historia de México) y del dossier "Juventudes étnicas contemporáneas en Latinoamérica" de la revista Cuicuilco, compilado por Maritza Urteaga y Luis F. García en 2015. 
${ }^{5}$ Para un estado del arte de la producción mexicana en esta línea, ver el artículo "El joven indígena en Chiapas: el re-conocimiento de un sujeto histórico" de Tania Cruz Salazar (2012).

${ }^{6}$ El término clivaje se usa también en las ciencias naturales. En geología alude a la tendencia de los minerales a dividirse en capas paralelas. En física de los materiales, el término refiere a los planos de quiebre de los cristales, asociados a la densidad de átomos de dichos planos y a la anisotropía del medio. Agradecemos a la Dra. María Teresa Causa por esta explicación.

${ }^{7}$ Castro, Rojas y Ruiz (2008) también mencionan a la juventud indígena como lugar de enunciación en el campo de las producciones artísticas en Chile.

${ }^{8}$ Según Kunin, la música opera como registro a través del cual los jóvenes critican a la clase política "tradicional", expresan su apoyo al presidente Evo Morales e intentan concientizar a otros jóvenes sobre diferentes cuestiones vinculadas a la ciudadanía.

${ }^{9}$ Por etnografía situada entendemos aquella que se centra en el estudio de comunidades locales y sus prácticas de resistencia o adaptación al capitalismo global (Marcus, 2001).

${ }^{10}$ Según Virtanen (2007), la participación en este campo otorga a los jóvenes manchineri, kaxinawá y apuriña nuevos "capitales simbólicos y sociales" que acaban modificando su habitus y poniendo en cuestión la imagen tradicional de los pueblos amazónicos como sistemas cerrados. Para Solís y Martínez (2015), el foco está en los modos prácticos en que un mismo joven universitario nahua se posiciona de maneras diferentes frente a las diversas identificaciones en el espacio social juvenil. Por su parte, Serrano Santos (2015) sostiene que los jóvenes indígenas de Chiapas configuran estilos de vida a partir de la etnia — representada por las experiencias y percepciones que mantienen respecto de la vida en la comunidad de origen y algunos rasgos culturales asociados a la categoría indígena- y la condición de juventud - representada en las experiencias y percepciones sobre su vida en la ciudad y los referentes simbólicos que los identifican como jóvenes en un contexto determinado-.
${ }^{11}$ Entre las investigaciones fundadas en la etnografía situada, quienes analizan casos en Chile manifiestan una preocupación más clara por la relación entre los jóvenes indígenas y el movimiento organizado en torno a la demanda de derechos ante el Estado.

${ }^{12}$ Los enfoques sustancialistas son aquellos que entienden que la etnicidad se define, y se mide, en términos de contenido cultural.

13 El contacto de los jóvenes con los espacios urbanos provoca, según este análisis, un proceso de transculturación en las comunidades que se manifiesta en transformaciones en los ritos de pasaje a la vida adulta y de matrimonio

${ }^{14}$ La perspectiva formalista desplaza la mirada de los contenidos culturales a las prácticas de interacción —tradición académica inaugurada por Fredrik Barth en 1969 y por Roberto Cardoso de Oliveira en 1971- Desde esa perspectiva, no resulta relevante formular la pregunta sobre el contenido cultural, sino analizar las prácticas de marcación y automarcación que transforman ciertos elementos en "diacríticos identitarios" en función de la interacción.

${ }^{15}$ Para un debate sobre la definición de juventud como etapa de transición en el caso de los pueblos indígenas, ver Pérez Ruiz (2008).

16 En el caso etnográfico que desarrolla, plantea que la misma idea de comunidad se va definiendo históricamente en el marco del conflicto agrario. El trabajo de Bello Maldonado retomalas teorías purépecha de la edad en tensión con las teorías occidentales, al tiempo que cuestiona la interpretación de la experiencia migratoria como efecto de la globalización.

${ }^{17}$ Así, por ejemplo, grupos desagregados étnicamente pueden estar agregados en términos de clase, y el clivaje de clase puede, al mismo tiempo, establecer desagregaciones al interior de un grupo étnico.

${ }^{18}$ En este sentido es en el que Maya Lorena Pérez Ruiz (2011) recupera la observación de Guillermo Bonfil Batalla en torno a la vinculación de la categoría "indígena" con la experiencia colonial y las relaciones de poder que instaura. Por su parte, en su libro La alteridad del cuarto mundo Claudia Briones (1998) desarrolla las críticas a la perspectiva formalista que someramente presentamos aquí. 


\section{Referencias bibliográficas}

Aguilera Aguila, Nelson y Blas Flores Aguila (2006). "Algunas consideraciones sobre los indígenas de Magallanes". En Observatorio de Juventud. Jóvenes y Pueblos Originarios, 3(12): 72-78.

Alvarado, Sara Victoria, Julián Loaisa y Jhoana Patiño (2010). "Jóvenes indígenas en Colombia. Acciones de resistencia: el caso del movimiento juvenil Álvaro Ulcué". Ponencia presentada en el Coloquio Internacional Juventud, Etnicidad, Ruralidad y Movimientos Translocales en Latino América, 21 y 22 de octubre, Bariloche, Argentina.

Ambriz Aguilar, Miriam Lisbeth (2011). Habitando fronteras: jóvenes purhépecha en la zona metropolitana de Guadalajara. Tesis de maestría, Centro de Investigaciones y Estudios Superiores en Antropología Social de Occidente, México.

Aravena Reyes, Andrea (2006). "Identidades étnicas, identidades sociales: la etnicidad de cara al siglo XXI (...a propósito de la identidad étnica en los jóvenes indígenas urbanos)". En Observatorio de Juventud. Jóvenes y Pueblos Originarios, 3(12): 5-16.

Barth, Fredrik (1976). "Introducción". En Fredrik Bartth (comp.), Los grupos étnicos y sus fronteras. La organización social de las diferencias culturales. México: Fondo de Cultura Económica, pp. 7-49.

Beckett, Jeremy (1988). Past and Present. The Construction of Aboriginality. Canberra: Aboriginal Studies Press.

Bello Maldonado, Álvaro (2008). "Los espacios de la juventud indígena. Territorio y migración en una comunidad purépecha de Michoacán, México". En Maya Lorena Pérez Ruiz (comp.), Jóvenes indígenas y globalizaciónen América Latina. México: INAH, pp. 161-179.

Bojórquez Chapela, Tiosha (2009). "Hip-hop, rap y spoken word como medios del patrimonio cultural inmaterial: una perspectiva desde el sur global". En Lourdes Arizpe (coord.), Compartir el patrimonio cultural inmaterial: narrativas y representaciones. México: CRIM/DGCP, pp. 249-269.

Briones, Claudia (1998). La alteridad del "Cuarto Mundo". Una deconstrucción antropológica de la diferencia. Buenos Aires: Ediciones del Sol.
Briones, Claudia (2005). "Formaciones de alteridad: contextos globales, procesos nacionales provinciales". En Claudia Briones (ed.), Cartografías argentinas: politicas indigenistas y formaciones provinciales de alteridad. Buenos Aires: Antropofagia, pp. 11-43.

Briones Claudia y Alejandra Siffredi (1989). "Discusión introductoria sobre los límites teóricos de lo étnico". En Cuadernos de Antropología, 3: 5-24.

Calfio Montalvo, Margarita (2006). "Pueblos indígenas y juventud en el siglo XXI: una mirada desde la diversidad y el género". En Observatorio de Juventud. Jóvenes y Pueblos Originarios, 3(12): 38-45.

Camus, Manuela (2008). “Los jóvenes 'indígenas' en la ciudad de Guatemala y la relatividad de la ideología". En Maya Lorena Pérez Ruiz (comp.), Jóvenes indígenas y globalización en América Latina. México: INAH, pp. 239-255.

Candia, Eduardo, Nivoska Damianovic y Tália Leibovitz (2006). "Juventudes indígenas: diagnóstico de integración en Chile". En Observatorio de Juventud. Jóvenes y Pueblos Originarios, 3(12): 17-28.

Cardoso De Oliveira, Roberto (1971). "Identidad étnica. Identificación y manipulación”. En América Indígena, XXX(4).

Castro Lucic, Milka, Gemma Rojas Roncagliolo y Carlos Ruiz Rodríguez (2008). "La juventud indígena en Chile: expectativas y demandas". En Maya Lorena Pérez Ruiz (comp.), Jóvenes indígenas y globalización en América Latina. México: INAH, pp. 93-112.

Citro, Silvia (2005). "Ritual y espectáculo en la música indígena: el caso de los jóvenes toba del Chaco argentino". En Latin American Music Review, 26(2): 318346.

Cruz Salazar, Tania (2015). “Experimentando California. Cambio generacional entre tzeltales y choles de la selva chiapaneca". En Cuicuilco, 22(62): 217-239.

Cruz Salazar, Tania (2012). "El joven indígena en Chiapas: el re-conocimiento de un sujeto histórico". En Liminar. Estudios Sociales y Humanísticos, X(2): 145-162.

Cuminao Rojo, Clorinda (2006). "Wekeche: identidad juvenil mapuche en la región metropolitana”. En Observatorio de Juventud. Jóvenes y Pueblos Originarios, 3(12): 58-65. 
De Rivero, Oscar (2009). "Ciudad e identidad cultural. ¿Cómo se relacionan con lo urbano los indígenas amazónicos peruanos en el siglo XXI?". En Bulletin de l'Institut Français D'Études Andines, 38(1): 47-59.

Del Popolo, Fabiana, Mariana López y Mario Acuña (2010). Juventud indígena y afrodescendiente en América Latina: inequidades sociodemográficas y desafíos de políticas. S.l.: CEPAL/UNFPA/OIJ.

Feixa, Carles y Yanko González Cangas (2006). "Territorios baldíos: identidades juveniles indígenas y rurales en América Latina". En Papers, 79: 171-193.

Fischer, Eva (2008). "Jóvenes y padres. Identidad, procesos de transformación y sociedad andina en Bolivia”. En Maya Lorena Pérez Ruiz (comp.), Jóvenes indígenas y globalización en América Latina. México: INAH, pp. 125-139.

García Álvarez, Luis Fernando (2015). “Nosotros integramos la forma de ver el mundo de nuestra comunidad y de la ciudad. La juventud mixteca en el Área Metropolitana de Monterrey, Nuevo León”. En Cuicuilco, 22(62): 241-264.

García Martínez, Ariel (2012). "Juventud indígena en el Totonacapan Veracruzano". En Liminar. Estudios Sociales y Humanísticos, 10(1): 75-88.

Grossberg, Lawrence (1996). "Identity and Cultural Studies: Is That All There Is?" En Stuart Hall y Paul Du Gay (eds.), Questions of Cultural Identity. Londres: Sage Publications.

Guerrero Jiménez, Bernardo (2007). "Identidad sociomusical de los jóvenes aymaras: la música sound". En Última Década, 27: 11-25.

Guevara Sánchez, Berenice (2015). "Pandillerosindígenas: el caso de la comunidad purépecha de Angahuan”. En Cuicuilco, 22(62): 193-216.

Igreja, Rebecca (2008). "Negociando identidades. La participación de los jóvenes en las organizaciones indígenas de la ciudad de México". En Maya Lorena Peréz Ruiz (comp.), Jóvenes indígenas y globalización en América Latina. México: INAH, pp. 219-238.

Kertzer, David (1978). "Review: Theoretical Developments in the Study of Age-Group Systems". En American Ethnologist, 5(2): 368-374.
Kropff, Laura (2005). “Activismo mapuche en Argentina: trayectoria histórica y nuevas propuestas”. En Pablo Dávalos (comp.), Pueblos indígenas, estado y democracia. Buenos Aires: CLACSO, pp. 103-132.

Kropff, Laura (201la). "Apuntes conceptuales para una antropología de la edad”. En Avá, Revista de Antropología, 16: $171-187$

Kunin, Johana (2009). "Algunas notas sobre el rap político del altiplano boliviano: mucho más que un caso 'exótico' de jóvenes indígenas que cantan ritmos estadounidenses". Ponencia presentada en el 53 Congreso Internacional de Americanistas, 19 al 24 de julio, México.

LlanosErazo, Daniel(2013). “Asimetríasy aproximaciones a la problemática educativa de jóvenes indígenas de la Sierra Central ecuatoriana”. En Ánfora, 19(34): 81-95.

Maidana, Carolina Andrea, María Adelaida Colangelo y Liliana Tamagno (2013). "Ser indígena y ser joven: entre la etnicidad y la clase”. En Desacatos, 42: 131-144.

Marcus, George (2001). "Etnografía en/del sistema mundo. El surgimiento de la etnografía multilocal". En Alteridades, 11(22): 111-127.

Mayorga, Martha Lidia y Marcela Marcia Bautista (2010). "Inclusión de los jóvenes indígenas y de municipios pobres de Colombia en la educación superior". Ponencia presentada en el Coloquio Internacional Juventud, Etnicidad, Ruralidad y Movimientos Translocales en Latino América, 21 y 22 de octubre, Bariloche.

Muñoz Millalonco, Manuel (2006). "Jóvenes y Autoridades tradicionales Williche: El encuentro en un nuevo camino para Chiloé". En Observatorio de Juventud. Jóvenes y Pueblos Originarios, 3(12): 29-37.

Negrín da Silva, Diana (2015). "Makuyeika: la que anda en muchas partes". En Cuicuilco, 22(62): 37-59.

Neto, Joaquim José (2004). Jovens tapuios do carretão: processos educativos de reconstrução de identidade indígena. Tesis de maestría, Universidade Católica de Goiânia, Brasil.

Ossola, María Macarena (2015). "Entre permisos y ejemplos. Reconfiguraciones familiares entre los jóvenes universitarios wichí en el noroeste argentino". En Cuicuilco, 22(62): 75-90. 
Pacheco Ladrón de Guevara, Lourdes (2009). “El olvido de los jóvenes rurales indios de la Sierra Madre Occidental de México". Ponencia presentada en el 53 Congreso Internacional de Americanistas, 19 al 24 de julio, México.

Padawer, Ana y Lucila Rodríguez Celín (2015). “Ser del monte, ser de la chacra: experiencias formativas e identificaciones étnicas de jóvenes rurales en el noreste argentino". En Cuicuilco, 22(62): 265-286.

Paladino, Mariana (2006). "Formas antiguas y actuales de ser joven' entre los ticuna de la región del Alto Amazonas brasileño: discursos moralizantes y sus ambigüedades". Ponencia presentada en el VIII Congreso Argentino de Antropología Social, Universidad Nacional de Salta, 19 al 22 de septiembre, Salta.

Pereira Mendes, Edmundo Marcelo (2011). “A construção da juventude para o Terena citadino (Campo Grande/ MS Brasil)". Ponencia presentada en el X Congreso Argentina de Antropología Social, 29 de noviembre y 2 de diciembre, Buenos Aires.

Pérez Ruiz, Maya Lorena (coord.) (2008). Jóvenes indígenas y globalización en América Latina. México: INAH.

Pérez Ruiz, Maya Lorena (2011). "Retos para la investigación de los jóvenes indígenas". En Alteridades, 2l(42): 65-75.

Rivas Toledo, Alexis (2008). "Los jóvenes indígenas en Ecuador: un ensayo de análisis demográfico, de representación y etnicidad". En Maya Lorena Pérez Ruiz (comp.), Jóvenes indígenas y globalización en América Latina. México: INAH, pp. 113-122.

Rodríguez Calderón de la Barca, Diego (2015). "Una exploración etnográfica sobre las y los jóvenes estudiantes y egresados de la UNICH, San Cristóbal de las Casas". En Cuicuilco, 22(62): 175-191.

Romer, Marta (2008). "Los hijos de migrantes indígenas en la ciudad de México. Problemas de identidad étnica". En Maya Lorena Pérez Ruiz (comp.), Jóvenes indígenas y globalización en América Latina. México: INAH, pp. 205-218.

Ruiz Lagier, Verónica (2008). "Refugio guatemalteco. Juventud, identidad y migración en La Gloria, Chiapas, México". En Maya Lorena Pérez Ruiz (comp.), Jóvenes indígenas y globalización en América Latina. México: INAH, pp. 181-202.
Serrano Santos, María Laura (2015). "'Soy de los dos lados, a la mitad me quedo'. Estilos de vida en jóvenes indígenas urbanos de San Cristóbal de las Casas, Chiapas". En Cuicuilco, 22(62): 149-173.

Stella, Valentina (2013). "De Kajfv malen a Werken: trayectoria de una joven mapuche-tehuelche". En Pasado Por-venir, 7(8), Trelew.

Solís Domínguez, Daniel y Consuelo Patricia Martínez Lozano (2015). “Género, sexualidad y cuerpo. Campo juvenil y jóvenes universitarios indígenas de San Luis Potosí". En Cuicuilco, 22(62): 121-148.

Terrazas Merino, Maziel (2008). “Jóvenes quechuas del ayllu Chari, La Paz, Bolivia: identidad, globalización, imaginarios y bienes culturales". En Maya Lorena Pérez Ruiz (comp.), Jóvenes indígenas y globalización en América Latina. México: INAH, pp. 141-160.

Tipa, Juris (2015). "Una aproximación a clase social, género y etnicidad en el consumo de música entre los estudiantes de la Universidad Intercultural de Chiapas". En Cuicuilco, 22(62): 91-112.

Unda Lara, René y Germán Muñoz (2011). "La condición juvenil indígena: elementos iniciales para su construcción conceptual”. En Última Década, 34: 35-50.

Urteaga Castro Pozo, Maritza (2009). "Retos contemporáneos en los estudios sobre juventud". Ponencia presentada en el 53 Congreso Internacional de Americanistas, 19 al 24 de julio, México.

Urteaga Castro Pozo, Maritza y Luis Fernando García Álvarez (coords.) (2015). "Juventudes étnicas contemporáneas en Latinoamérica”. En Cuicuilco, 22(62): 9-35.

Valladares De La Cruz, Laura Raquel (2008). "Ser mujer y ser joven en las comunidades indígenas de México". En Maya Lorena Pérez Ruiz (comp.), Jóvenes indígenasy globalizaciónen América Latina. México: INAH, pp. 69-92.

Virtanen, Pirjo Kristina (2007). "O novo habitus de jovens indígenas e as relações interetnicas na amazonia urbana”. En Anales Nueva Epoca, 9. Disponible en: http://gupea. ub.gu.se/dspace/bitstream/2077/4496/1/anales_9-10_ virtanen.pdf.

Vitti, Vaneska Taciana y Carmen Junqueira (2015). "Jovens Kamaiurá no século XXI". En Cuicuilco, 22(62): 61-73. 
Zapata Cardona, Carlos Andrés y Mauricio Hoyos Agudelo (2005). "¿Existe una condición de juventud indígena?" En Nómadas, Jóvenes Contemporáneos: entre la Heterogeneidad y las Desigualdades, 23: 28-37.
Zebadúa, Juan Pablo (2009). "Cultura e identidades juveniles en el Totonacapan: la construcción transcultural de jóvenesindígenas". Ponencia presentada en el 53 Congreso Internacional de Americanistas, 19 al 24 de julio, México.

\section{Cuadro 1. Una pequeña caja de herramientas}

\section{Enfoques generales}

1) Edad y etnicidad como datos dados

2) Juventud como dato + Etnicidad como problema

3) Etnicidad como dato + Juventud como problema

4) Edad y etnicidad como problemas

\section{Perspectivas}

Demografía y estadísticas en general

Lugares de enunciación

Etnografía situada

Sustancialismo étnico

Formalismo étnico

Teorías académicas de la edad

Teorías sociales de la edad

Sustancialismo étnico + Formalismo etario

Juventud y etnicidad como condiciones de un sujeto

Edad y etnicidad como clivajes

Fuente: elaboración propia. 